\title{
A low-cost device for measuring local magnetic anomalies in volcanic terrain
}

\author{
Bertwin M. de Groot ${ }^{1,2}$ and Lennart V. de Groot $^{1}$ \\ ${ }^{1}$ Paleomagnetic laboratory Fort Hoofddijk, Utrecht University, Budapestlaan 17, 3584 CD Utrecht, the Netherlands \\ ${ }^{2}$ Technical \& Analytical Support Earth Sciences, Utrecht University, Princetonlaan 8a, 3584 CB Utrecht, the Netherlands
}

Correspondence: Bertwin M. de Groot (b.m.degroot@uu.nl)

Received: 22 August 2018 - Discussion started: 15 November 2018

Revised: 1 July 2019 - Accepted: 11 July 2019 - Published: 22 August 2019

\begin{abstract}
Reconstructions of the past behavior of the geomagnetic field critically depend on the magnetic signal stored in extrusive igneous rocks. These rocks record the Earth's magnetic field when they cool and retain this magnetization on geological timescales. In rugged volcanic terrain, however, the magnetic signal arising from the underlying flows may influence the ambient magnetic field as recorded by newly formed flows on top. To measure these local anomalies in the Earth's magnetic field directly we developed a low-cost field magnetometer based on a fluxgate sensor. To improve the accuracy of the obtained paleomagnetic vector and user-friendliness of the device, we combined this fluxgate sensor with tilt and GPS sensors to rotate the measured magnetic vector to true north, east, and down. The data acquisition is done using a ruggedized laptop, and data are immediately available for first-order interpretation. The first measurements done on Mt. Etna show local variations in the ambient magnetic field that are larger than expected and illustrate both the accuracy (certainly $<0.5^{\circ}$ in paleomagnetic direction) and potential of our new device.
\end{abstract}

\section{Introduction}

The Earth's magnetic field has a pivotal role in the Earth sciences and has applications in magnetostratigraphy, tectonics, and studies of the deep Earth. Furthermore, the Earth's magnetic field protects us against electromagnetically charged particles from the Sun that, if they were not deflected by the Earth's magnetic field, could slowly strip away our atmosphere. An excess of such charged particles interferes with technological advancements such as wireless communication and satellites. Over the past centuries the Earth's magnetic field has lost more than $20 \%$ of its strength, and regionally variations are even more dramatic (e.g., Pavón-Carrasco et al., 2014; Nilsson et al., 2014). To come to a thorough understanding of the behavior of the Earth's magnetic field it is paramount to have a record of the behavior of the Earth's magnetic field through (geologic) time and for different locations. The only recorders of the Earth's magnetic field available all over the globe and throughout geologic history are extrusive volcanic rocks, e.g., lava. Lava becomes magnetic when the iron-oxide-bearing minerals cool trough their Curie temperature and stores this magnetization even on geological timescales. By sampling many cooling units with a known age in a volcanic edifice it is possible to reconstruct regional variations in the Earth's magnetic field for a certain region, while its resolution in time is determined by the availability of well-dated cooling units (e.g., de Groot et al., 2013a; Greve et al., 2017).

The methodologies of obtaining paleodirections and paleointensities from a single cooling unit have been tested by sampling recent flows, e.g., flows that acquired their magnetization in a known magnetic field (e.g., Biggin et al., 2007; de Groot et al., 2013b). The paleointensity proves to be especially hard to reconstruct, and often experiments that are deemed "technically successful" produce underestimates or overestimates of the known paleofield. Furthermore, paleodirections are sometimes hard to obtain reliably (e.g., Castro and Brown, 1987; Coe et al., 2014). Often, the reasons for these deviations are sought in rock-magnetic processes such as "thermal alteration" or "multidomain effects" that are known to hamper paleomagnetic experiments, but it is also possible that these deviations from the expected intensities 
and directions actually arise from local magnetic anomalies caused by the magnetization of underlying lava flows. Local anomalies are known to cause deviations in magnetic compass readings in volcanic terrain, and they may therefore very well influence the magnetic field as recorded by lavas when they cool (Baag et al., 1995; Valet and Soler, 1999; Tanguy and Le Goff, 2004).

Here, we present a low-cost device that measures the ambient magnetic field at a selectable distance from the surface of a lava flow to enable systematic mapping of local magnetic anomalies in volcanic terrain: the AnomalyMapper. Its design revolves around a three-axis fluxgate sensor that is mounted on an aluminum frame. To determine the declination, inclination, and intensity of the ambient magnetic field, we need to know the orientation of the fluxgate sensor with respect to true (geographic) north, east, and down. To this end, there are two main hurdles to overcome: (1) it is impossible to align the fluxgate sensor perfectly along the vertical while measuring in volcanic terrain, and (2) we cannot use a magnetic compass to orient the fluxgate to true north, as we are measuring local magnetic anomalies that interfere with compass readings. During normal operation it is possible to keep the AnomalyMapper upright within $\pm 3^{\circ}$ of true vertical by using a bubble level. To also correct for the remaining deviation from vertical, we use an accelerometer (e.g., tilt sensor) that is fixed to the fluxgate sensor to determine the orientation of the AnomalyMapper with respect to the direction of gravity; these measurements are used to rotate the fluxgate measurements to true vertical. An intuitive way to avoid using a magnetic compass would be to use a Sun compass, but this would render the AnomalyMapper useless when the sky is overcast. We therefore use a scope to orient the AnomalyMapper to a fixed reference point on the ground with a known (GPS) location. By logging the position of the AnomalyMapper for each measurement with a highly accurate GPS sensor we can determine the bearing of the measurement location to the reference point and hence rotate the measurements to true north and east. This experimental design yields highly accurate magnetic measurements, while the measurements can be done quickly in the field.

To test the performance of the AnomalyMapper, we mapped local magnetic anomalies nearby and on top of a block of lava from the 2002 flow of Mt. Etna (Sicily, Italy) at three distances above the ground. Furthermore, we assess the performance of the correction based on the tilt sensor to rotate the fluxgate measurements to true vertical during normal operation as well as under rather extreme circumstances in which the AnomalyMapper was held under angles up to $25^{\circ}$ from true vertical.

Our AnomalyMapper is a low-cost device, and many parts are likely to be readily available in paleomagnetic laboratories. Apart from the fluxgate sensor that is commercially available for EUR $\sim 2000$, the setup totals EUR $<1500$, including a ruggedized laptop suitable for use in the field, a tilt

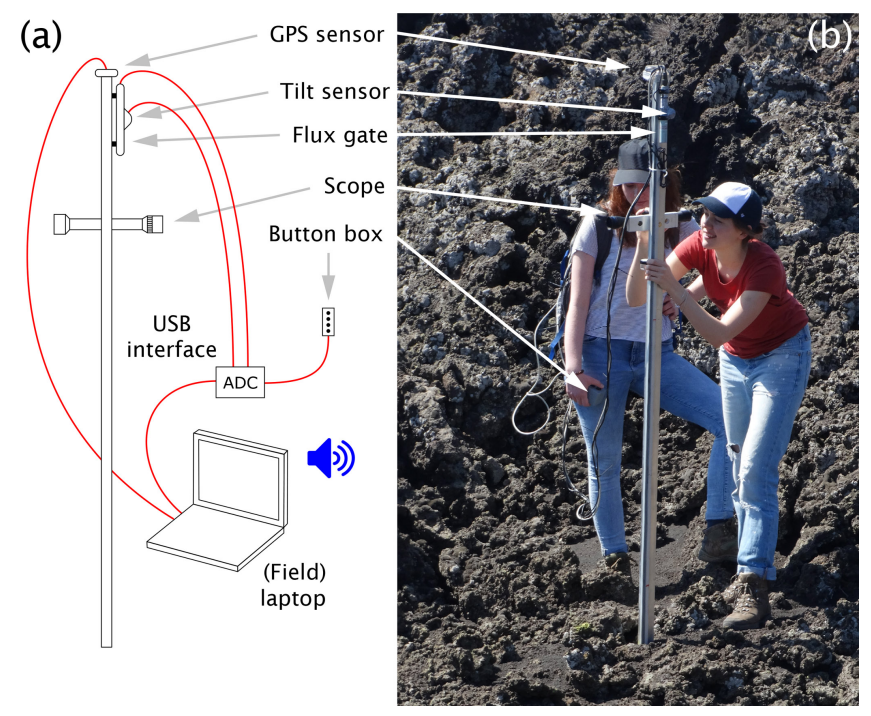

Figure 1. The AnomalyMapper. The AnomalyMapper consists of a GPS sensor, a tilt sensor, a fluxgate sensor, and a scope mounted on an aluminum frame, and it is operated by pressing buttons on a button box (a). The electronic components are connected (red lines) to a ruggedized laptop through USB interfaces, and the laptop has audible feedback to the users. The AnomalyMapper is easily operated by two people in the field (b), while the USB interface and laptop are carried in a small backpack.

sensor, interfaces between analog sensors and the laptop, and all other hardware necessary to build the instrument.

\section{Physical description}

The backbone of the AnomalyMapper is a rectangular aluminum tube with dimensions $40 \times 40 \times 2000 \mathrm{~mm}$ (Fig. 1a). An aluminum I profile was glued along its entire length, allowing the fluxgate sensor to slide from top to bottom using aluminum mounts (in which the stainless-steel bolts were changed to brass ones). The tilt sensor was glued to the fluxgate sensor to ensure an assessment of the actual orientation of the fluxgate sensor with respect to gravity. The GPS sensor was mounted at the top of the frame so that it is not obscured while the AnomalyMapper is in use. The scope (Lensolux 3$9 \times 32$, with crosshair) was fixed onto an aluminum profile and bolted to the frame using brass nuts, washers, and bolts. About halfway along the frame a two-dimensional bubble level was fixed to the frame using a small piece of aluminum profile (Fig. 1a).

\subsection{GPS sensor}

The GPS sensor is a vital part of the AnomalyMapper as the rotation towards true north and east depends on the accuracy of its known position. Here we use a commercially available Navilock 6004P MD6 based on a u-blox NEO-6P chip set. It has a horizontal accuracy of $<1 \mathrm{~m}$ and a vertical accuracy 
of $<2 \mathrm{~m}$. This sensor directly connects with the ruggedized laptop through its USB interface. When starting a series of measurements the GPS sensor needs some time to acquire enough satellite fixes to provide an accurate location. This "cold start" is specified as $38 \mathrm{~s}$ but may be longer in practice. During a series of measurements the GPS sensor is read continuously to ensure that its reading is accurate $1 \mathrm{~s}$ after positioning the AnomalyMapper (hot start). During normal operation this $1 \mathrm{~s}$ is needed to position and aim the AnomalyMapper to the reference point. The time needed to acquire an accurate GPS position of the AnomalyMapper is therefore not restrictive during normal use in the field.

\subsection{Tilt sensor}

The orientation of the AnomalyMapper with respect to gravity is measured by a three-axis accelerometer chip; here we choose an Analog Devices ADXL335 chip on a SparkFun breakout board (SEN-09269). The ADXL335 is a microelectromechanical system (MEMS) device with sensitivity corresponding to up to $0.1^{\circ}$, albeit with less than ideal drift characteristics and offset as well as sensitivity accuracy that require calibration and correction. Offset and sensitivity calibration values were established in the lab, and drift correction values are calculated for each measurement session. This chip is powered from the laptop using a Seeed step-down DC power converter based on an MP1584 chip from Monolithic Power Systems. The power supply voltage provided to the ADXL335 is measured simultaneously with each readout, as the three analog accelerometer outputs are ratiometric to the power supply. Identical $1 \mathrm{~Hz}$ bandwidth resistorcapacitor (RC) low-pass filters are used on all four channels for increased noise reduction and accurate recording of the power supply voltage.

\subsection{Fluxgate sensor}

We used a commercially produced fluxgate sensor that was readily available in our paleomagnetic laboratory: the Bartington Mag-03MCES100 connected to a Bartington power supply and display unit. This fluxgate has a dynamic range of 0 to $100 \mu \mathrm{T}$, which is well suited to measure the range of expected field intensities in volcanic terrain. It has a threeaxis analog output, so the precision of the measurements is determined by the analog-digital (AD) converter used; we chose a 16-bit AD converter, leading to an effective precision of $\ll 25 \mathrm{nT}$.

\subsection{Interfacing and computer}

The analog outputs from the tilt and fluxgate sensors are connected to a USB data acquisition (DAQ) device; here we used a Measurement Computing USB-1608G. In the field the main user interface is a handheld button box with four buttons. Each button gives a label (1-4) to the measured data, so the four buttons can be used to measure at four different dis- tances above the ground or to label repeated measurements at the same location. The button box is connected to digital inputs on the USB-1608G. A ruggedized Lenovo laptop runs the data collection software, requiring no user input in the field after initialization.

\subsection{Software}

The data collection software is written in LabVIEW 2017. The software continuously collects GPS data and, when the operator presses a button, records data points for the fluxgate and tilt sensor. Data acquisition is simultaneous for all channels at a $10 \mathrm{ksps}$ sampling rate for 1000 samples per analog channel per measurement. The mean values of these $100 \mathrm{~ms}$ measurements are written into a .csv file along with the GPS position for that measurement location and the height of the measurement according to the button pressed. User feedback is an audible confirmation of a successful data point recording or an audible warning when the GPS data are old or the fluxgate or tilt sensor data are outside expected bounds.

\section{Data acquisition}

The AnomalyMapper is easily operated by two people (Fig. 1b). The first aims the AnomalyMapper at the reference point by looking through the scope, while the second keeps the AnomalyMapper more or less upright by keeping an eye on the bubble level and acquires data by pushing the appropriate button on the button box. Aiming the AnomalyMapper at the reference target needs to be done with great care, as this orientation defines the measured declination of the magnetic field. If measurements are to be done at different heights above the ground, it is easiest to use spray paint to indicate the measurement locations and follow the same section as many times as necessary instead of moving the fluxgate along the frame of the AnomalyMapper multiple times per location.

\section{Data processing and reference frames}

The magnetic flux densities as measured by the fluxgate must be rotated towards north, east, and down to be informative on the full vector of the Earth's magnetic field in a particular location. To this end four rotations are necessary: (1) align the tilt sensor measurements to the reference frame of the AnomalyMapper, (2) align the fluxgate measurements to the reference frame of the AnomalyMapper, (3) rotate the $z$ axis of the fluxgate measurements to vertical based on the tilt sensor measurements while preserving the orientation of its $x$ axis to the direction of the reference point, and (4) rotate the measured magnetic flux densities towards north, east, and down around the $z$ axis of the AnomalyMapper.

Geosci. Instrum. Method. Data Syst., 8, 217-225, 2019 


\subsection{Aligning tilt sensor measurements to the AnomalyMapper's frame}

The tilt sensor is attached to the fluxgate such that gravity during normal (upright) use is distributed over the three axes of the sensor so that each axis performs optimally. To rotate the tilt sensor measurements to the coordinate system as defined by the frame of the AnomalyMapper ( $x$ in the direction of the scope, $y$ to the right of the scope, and $z$ downwards along the rod; Fig. 2a), we define a rotation matrix. This rotation matrix is created with the readouts of the tilt sensor when the AnomalyMapper is successively oriented with its $x$ (top row), $y$ (middle row), and $z$ (bottom row) axes aligned with gravity. This yields the following rotation matrix for the tilt sensor, $\mathbf{G}_{\mathrm{f}}$, in which the first character of the indices denotes which axis of the AnomalyMapper was aligned with gravity and the second character indicates the axis of the tilt sensor:

$\mathbf{G}_{\mathrm{f}}=\left[\begin{array}{lll}g_{x x} & g_{x y} & g_{x z} \\ g_{y x} & g_{y y} & g_{y z} \\ g_{z x} & g_{z y} & g_{z z}\end{array}\right]$.

The accuracy of the tilt sensor is affected by drift due to, e.g., temperature differences, but the precision within a limited time span under constant conditions is very good (Sect. 2.2). To correct for this absolute drift between different sites and the moment the rotation matrix $\mathbf{G}_{\mathrm{f}}$ was determined, we average all measurements done at one site (usually $\gg 100$ measurements) and assume that the average of these measurements represents the true vertical, i.e., has the same orientation as unit vector [001]. The vector of the averaged tilt sensor measurements in its $x, y$, and $z$ directions should therefore be equal to the bottom row of $\mathbf{G}_{\mathrm{f}}$. Differences between these two vectors arise from drift, and the measured data should be corrected for this before rotation matrix $\mathbf{G}_{f}$ can be used to align the tilt sensor data to the reference frame defined by the AnomalyMapper. Hence we define a correction vector $(\boldsymbol{\Delta} \boldsymbol{g})$ as the difference between the averaged measurements in the $x, y$, and $z$ axes of the tilt sensor obtained at one site (and within a couple of hours) and the bottom row of rotation matrix $\mathbf{G}_{\mathbf{f}}$ :

$\boldsymbol{\Delta} \boldsymbol{g}=\left[\begin{array}{l}\overline{g_{x}} \\ \overline{g_{y}} \\ \overline{g_{z}}\end{array}\right]-\left[\begin{array}{l}g_{z x} \\ g_{z y} \\ g_{z z}\end{array}\right]$.

The tilt sensor data can now be rotated to the reference frame of the AnomalyMapper by correcting a measured tilt vector $\left(\boldsymbol{g}_{\mathrm{m}}\right)$ for drift and multiplying it by the inverse of the rotation matrix:

$\boldsymbol{g}=\left(\boldsymbol{g}_{\mathrm{m}}-\boldsymbol{\Delta} \boldsymbol{g}\right) \cdot \mathbf{G}_{\mathrm{f}}^{-1}$.

\subsection{Aligning fluxgate measurements to the AnomalyMapper's frame}

Although the fluxgate was carefully aligned to the frame of the AnomalyMapper, a small, fortuitous misalignment could not be avoided. It is possible to rotate the fluxgate measurements to the reference frame defined by the AnomalyMapper using a similar rotation matrix as used for the tilt sensor, i.e., by creating a matrix with fluxgate readouts while applying a magnetic field successively in the three orthogonal axes. Since the $z$ axis of the fluxgate is perfectly aligned with the $z$ axis of the AnomalyMapper due to its construction, however, we choose to carefully measure the deviation of the $x$ and $y$ axes of the fluxgate with respect to the coordinate system of the AnomalyMapper ( $\alpha$, Fig. $2 b$ ) with a protractor and rotate the fluxgate measurements around its $z$ axis. This implies the use of the following rotation matrix $\mathbf{B}_{\mathrm{f}}$; due to the alignment of the $x, y$, and $z$ axes with respect to the AnomalyMapper this rotation is in the negative direction (using an angle $-\alpha$ ):

$\mathbf{B}_{\mathrm{f}}=\left[\begin{array}{ccc}\cos (-\alpha) & -\sin (-\alpha) & 0 \\ \sin (-\alpha) & \cos (-\alpha) & 0 \\ 0 & 0 & 1\end{array}\right]$.

The measured fluxgate data can now be rotated to the reference frame of the AnomalyMapper by multiplying a measured vector $\boldsymbol{B}_{\mathrm{m}}$ by this rotation matrix:

$\boldsymbol{B}_{\mathrm{f}}=\boldsymbol{B}_{\mathrm{m}} \cdot \mathbf{B}_{\mathrm{f}}$.

\subsection{Putting the fluxgate measurements upright}

While using the AnomalyMapper in the field great care is taken to position the stick upright; since the AnomalyMapper is handheld, however, deviations of up to $\pm 3^{\circ}$ are common. With the data of the tilt sensor we can rotate the fluxgate data to an upright reference frame with its $z$ axis vertical (Fig. 2cd), but we have to be careful to preserve the orientation of the $x$ axis of the AnomalyMapper towards the reference point. To this end we apply two rotations, the first around the $x$ axis of the new upright reference frame $(\phi)$ and the second around its $y$ axis $(\theta)$. Due to the alignment of the $x, y$, and $z$ axes the rotation around the $x$ axis is in the negative direction (using an angle $-\phi$ ), and the second is in the positive direction (using an angle $+\theta$ ). The angles $\phi$ and $\theta$ are defined as

$\phi=\arctan \left[\frac{g_{y}}{g_{z}}\right]$

and

$\theta=\arctan \left[\frac{g_{x}}{g_{z}^{\prime}}\right]$,

with $g_{x}, g_{y}$, and $g_{z}$ the tilt sensor data with respect to the frame of the AnomalyMapper (i.e., vector $g$ ), and $g_{z}^{\prime}$ the value of the $z$ axis of the tilt sensor data after the rotation 
(a)

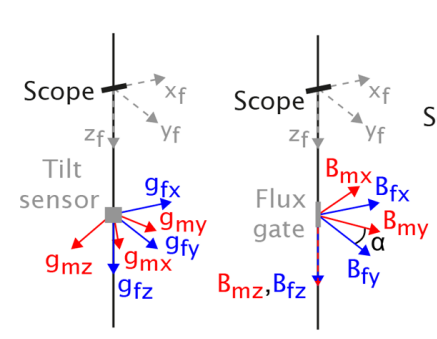

(c)

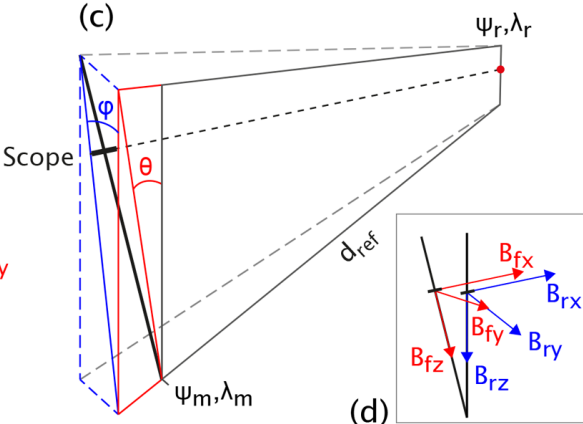

(e)

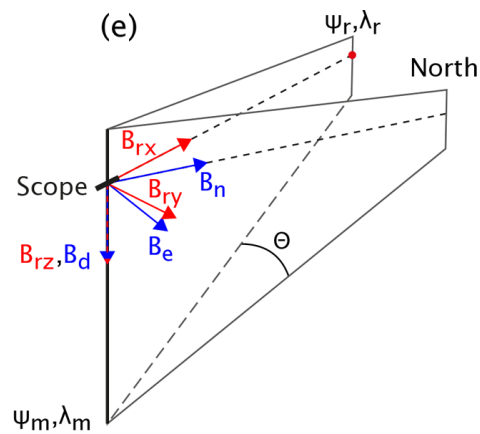

Figure 2. The different coordinate systems of the AnomalyMapper; in each panel the rotations are from the red to the blue coordinate systems. The axes of the tilt sensor $\left(g_{\mathrm{m} x}, g_{\mathrm{m} y}, g_{\mathrm{m} z}\right)$ are rotated to the axes defined by the frame of the AnomalyMapper $\left(g_{\mathrm{f} x}, g_{\mathrm{f} y}, g_{\mathrm{f} z}\right)(\mathbf{a})$, and the axes of the fluxgate $\left(B_{\mathrm{m} x}, B_{\mathrm{m} y}, B_{\mathrm{m} z}\right)$ are rotated over angle $\alpha$ around the $z$ axes of the AnomalyMapper to $B_{\mathrm{f} x}, B_{\mathrm{f} y}, B_{\mathrm{f} z}(\mathbf{b})$. Then the tilt sensor measurements are used to rotate the fluxgate measurements in the system of the AnomalyMapper $\left(B_{\mathrm{f} x}, B_{\mathrm{f} y}, B_{\mathrm{f} z}\right)$ over angles $\phi$ and $\theta$ to the reference frame, with $B_{\mathrm{r} x}$ pointing in the direction of the reference point (red dot; $\left.\left(\Psi_{\mathrm{r}}, \lambda_{\mathrm{r}}\right)\right) ; B_{\mathrm{r} y}$ and $B_{\mathrm{r} z}$ are vertical $(\mathbf{c}, \mathbf{d})$. The last rotation is over angle $\Theta$ (the bearing from the measurement location $\left(\Psi_{\mathrm{m}}, \lambda_{\mathrm{m}}\right)$ to the reference point) around the vertical axis to align $B_{\mathrm{r} x}$ to geographic north $\left(B_{\mathrm{n}}\right)$ and $B_{\mathrm{r} y}$ to geographic east $\left(B_{\mathrm{e}}\right)(\mathbf{e})$.

around the $x$ axis of the new reference frame. The rotation matrices associated with these rotations are

$\mathbf{R}_{1}=\left[\begin{array}{ccc}1 & 0 & 0 \\ 0 & \cos (-\phi) & -\sin (-\phi) \\ 0 & \sin (-\phi) & \cos (-\phi)\end{array}\right]$

and

$\mathbf{R}_{2}=\left[\begin{array}{ccc}\cos (\theta) & 0 & \sin (\theta) \\ 0 & 1 & 0 \\ -\sin (\theta) & 0 & \cos (\theta)\end{array}\right]$.

To rotate the data to the reference system defined by the reference point and the vertical a multiplication of vector $\boldsymbol{B}_{\mathrm{f}}$ with the two rotation matrices is sufficient:

$\boldsymbol{B}_{\mathrm{r}}=\boldsymbol{B}_{\mathrm{f}} \cdot \mathbf{R}_{1} \cdot \mathbf{R}_{2}$.

\subsection{Rotating fluxgate measurements towards true north}

The final step of the data processing is to rotate the fluxgate data to true north using the locations of the reference point and the AnomalyMapper (Fig. 2e). To this end we have to determine the bearing $(\Theta)$ from the measurement location (i.e., the location of the AnomalyMapper) to the reference point based on their GPS locations. Here we define the following: $\psi_{\mathrm{m}}$ and $\lambda_{\mathrm{m}}$ are the latitude and longitude of the measurement location, and $\psi_{\mathrm{r}}$ and $\lambda_{\mathrm{r}}$ are the latitude and longitude of the reference point. The bearing from the location of the measurement to the reference point with respect to true north is then given by

$$
\begin{aligned}
& \Theta=\arctan \\
& {\left[\frac{\sin \left(\lambda_{\mathrm{r}}-\lambda_{\mathrm{m}}\right) \cdot \cos \left(\psi_{\mathrm{r}}\right)}{\cos \left(\psi_{\mathrm{m}}\right) \cdot \sin \left(\psi_{\mathrm{r}}\right)-\sin \left(\psi_{\mathrm{m}}\right) \cdot \cos \left(\psi_{\mathrm{r}}\right) \cdot \cos \left(\lambda_{\mathrm{r}}-\lambda_{\mathrm{m}}\right)}\right] .}
\end{aligned}
$$

To rotate the vector $\boldsymbol{B}_{\mathrm{r}}$ to geographic coordinates we define the following rotation matrix to rotate over an angle $-\Theta$ around the vertical axis,

$\mathbf{R}_{3}=\left[\begin{array}{ccc}\cos (-\Theta) & -\sin (-\Theta) & 0 \\ \sin (-\Theta) & \cos (-\Theta) & 0 \\ 0 & 0 & 1\end{array}\right]$,

and multiply $\boldsymbol{B}_{\mathrm{r}}$ with this matrix:

$\boldsymbol{B}=\boldsymbol{B}_{\mathrm{r}} \cdot \mathbf{R}_{3}$.

\section{Experimental results}

To assess the performance of the AnomalyMapper we mapped magnetic anomalies on a roadcut in the 2002 flow of Mt. Etna $\left(15.7957^{\circ} \mathrm{N}, 15.0620^{\circ} \mathrm{E}\right)$. The anomalies were measured at 5,100, and $180 \mathrm{~cm}$ above the ground, and we used a traffic sign approximately $200 \mathrm{~m}$ down the road as a reference point. We measured a grid of $10 \times 11$ points in a rectangle of approximately $20 \times 22 \mathrm{~m}$. The road and rock face are roughly north-south. In each east-west line, the easternmost four data points are on the road, then two or three points are next to the rock face, and the remaining four to five points are on top of the outcrop (Fig. 3a, b). The elevation was measured by the GPS sensor; although the accuracy of the GPS sensor (vertically $<2 \mathrm{~m}$ ) does not necessarily allow for the mapping of the elevation of the outcrop properly, the main structures are produced very well (Fig. 3c).

The local variations in declination, inclination, and intensity are mapped in contour plots. The local anomalies are much more prominent at $5 \mathrm{~cm}$ above the ground and become smoother at 100 and $180 \mathrm{~cm}$ above the ground (Fig. 3). The magnetic field is more homogenous above the road, although the road is built on volcanic rock as well. Some features of the magnetic field correlate closely with the topography of 

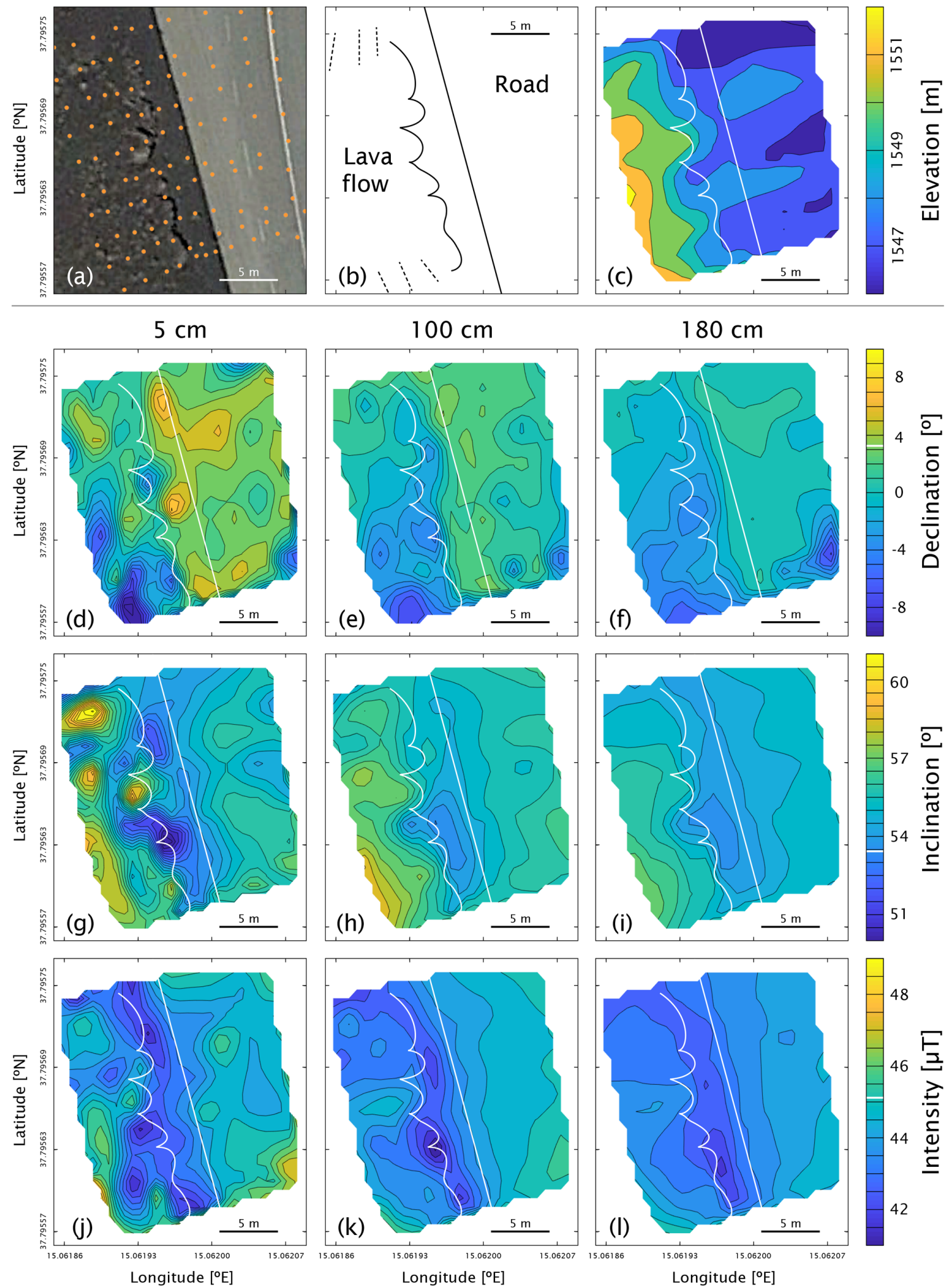

Figure 3. Experimental results as acquired on a roadcut in the 2002 flow of Mt. Etna. The GPS locations of the measurements are orange dots on a Google Earth image (a). The road, rock face (wavy solid line), and shallower north and south slopes of the lava flow (dashed lines) are sketched (b); the rock face and edge of the road are indicated in white in the other panels. The elevation was determined by the GPS sensor (c). The declinations (d-f), inclinations (g-i), and intensities (j-l) were measured at $5(\mathbf{d}, \mathbf{g}, \mathbf{j}), 100(\mathbf{e}, \mathbf{h}, \mathbf{k})$, and $180 \mathrm{~cm}(\mathbf{f}, \mathbf{i}, \mathbf{l})$ above the ground. For ease of comparison between panels with the same parameter the color schemes and contour lines are kept constant. The declination, inclination, and intensity as predicted by the International Geomagnetic Reference Field (IGRF, April 2018) are indicated as solid white lines in the scale bars. 
the rock face, e.g., the positive $\left(37.79567^{\circ} \mathrm{N}, 15.06193^{\circ} \mathrm{E}\right)$ and negative $\left(37.79563^{\circ} \mathrm{N}, 15.06195^{\circ} \mathrm{E}\right)$ anomalies in inclination at $5 \mathrm{~cm}$ above the ground (Fig. $3 \mathrm{~g}$ ) and the low intensities at $100 \mathrm{~cm}$ above the ground at $37.79563^{\circ} \mathrm{N}, 15.06195^{\circ} \mathrm{E}$ (Fig. 3k). Other anomalies at $5 \mathrm{~cm}$ above the ground may also be due to the influence of loose boulders or rocks on top of the lava flow that were easily $>30 \mathrm{~cm}$ in diameter (Fig. 3d, $g, \mathrm{j})$.

\section{Discussion}

\subsection{Experience in the field}

The AnomalyMapper is portable, suitable for air travel, and easy to use in the field. The acquisition of all data in Fig. 3 took less than $2.5 \mathrm{~h}$. Most parts of the AnomalyMapper are (or can be built as) water resistant, so with proper precautions to protect the ruggedized laptop and the $\mathrm{I} / \mathrm{O}$ device against rain it is possible to use the AnomalyMapper in most weather conditions.

The AnomalyMapper is most efficiently operated with two people: one aiming the AnomalyMapper at the reference point, while the other keeps the AnomalyMapper more or less upright by looking at the bubble level. When the magnetic anomalies are to be measured at more than one height above the ground it is most efficient to use spray paint to mark the measurement locations and return to these points after adjusting the height of the fluxgate.

\subsection{Accuracy and performance}

The tilt sensor is an important part of the design of the AnomalyMapper, as it enables accurate measurements when the AnomalyMapper is not exactly aligned with true vertical. To assess the performance of the tilt sensor we did 12 measurements in front of the paleomagnetic laboratory Fort Hoofddijk at Utrecht University $\left(52.08808^{\circ} \mathrm{N}, 5.17016^{\circ} \mathrm{E}\right)$ and processed the data with and without the tilt sensor correction in the spring of 2018. In June 2019, we obtained a reference measurement of the Earth's magnetic field at the same location using a horizontal surface that had an accuracy of $0.03^{\circ}$. The obtained reference values for the declination, inclination, and intensity were $-3.3^{\circ}, 65.5^{\circ}$, and $47.9 \mu \mathrm{T}$, respectively. Since the intensity measurements are not affected by the position of the AnomalyMapper we can compare the averaged measured intensities with their 1 standard deviation $(48.1 \pm 0.03 \mu \mathrm{T})$ directly to the reference field: the measured intensity is very close to the intensity value measured 1 year later but slightly higher.

During normal operation the AnomalyMapper can be kept within $<3^{\circ}$ of true vertical using the bubble level (Fig. 4a and $\mathrm{b}$, left). Before tilt correction the declination (with its 1 standard deviation) is $-0.8 \pm 1.2^{\circ}$ and the inclination is $65.8 \pm 0.3^{\circ}$. After tilt correction the declination and inclination become $-3.6 \pm 0.5$ and $65.2 \pm 0.1^{\circ}$, respectively. Both
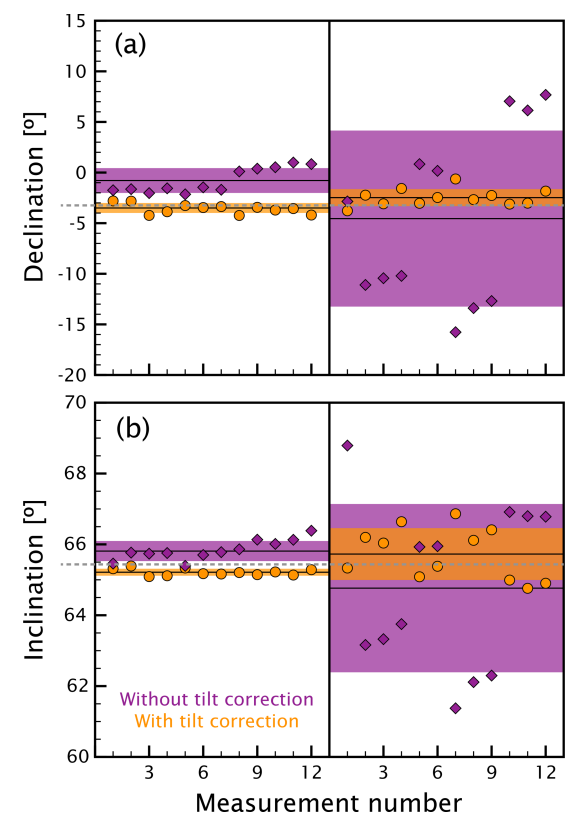

Figure 4. Comparison of the declination (a) and inclination (b) before and after tilt correction. We did 12 measurements while carefully positioning the AnomalyMapper upright using the bubble level (deviation form vertical $<3^{\circ}$, left-hand side of both panels) and 12 measurements with the AnomalyMapper more or less upright (deviation form vertical up to $25^{\circ}$, right-hand side of both panels). Each measurement is plotted with (orange circles) and without (purple diamonds) tilt correction; the averages of groups of 12 measurements are given as horizontal black lines with their associated 1 standard deviation intervals as shading in the corresponding color. The gray dashed lines are the independently measured values of the Earth's magnetic field.

the declination and inclination are only $0.3^{\circ}$ off the reference values. It must be emphasized that the reference values were measured 1 year later, and slight deviations may be explained by changes in the ambient field, changes in the Earth's magnetic field, or anthropogene contributions to the ambient magnetic field.

We then repeated the 12 measurements but allowed the AnomalyMapper to deviate up to $25^{\circ}$ from true vertical (Fig. 4a and b, right). Before tilt correction this yielded an average declination of $-4.6 \pm 8.7$ and an inclination of $64.6 \pm 2.4^{\circ}$. After correction using the tilt sensor data the declination and inclination became $-2.5 \pm 0.8$ and $65.7 \pm 0.7^{\circ}$, respectively. Again, the tilt sensor corrects the obtained declinations and inclinations to values closer to the reference measurement and, more importantly, to values closer to those obtained by keeping the AnomalyMapper $<3^{\circ}$ of true vertical. The deviations from the reference field are $0.8^{\circ}$ for the declination and $0.2^{\circ}$ for the inclination.

It is reassuring to note that the tilt sensor correction reduces the standard deviation associated with the declinations and inclinations dramatically; this implies that the mea- 
sured values converge towards their mean after tilt correction. Moreover, the declinations and inclinations for the measurements done with the AnomalyMapper are within $<3^{\circ}$ with deviations up to $25^{\circ}$; these are pretty close, further testifying to the improvements in accuracy using the tilt sensor.

\subsection{Temperature dependence of electronic components}

The performance of the electronic parts of the AnomalyMapper is temperature dependent, and it is therefore important to consider the temperature coefficients of the GPS, fluxgate, tilt sensor, and DAQ devices. The Navilock 6004P MD6 GPS sensor is built for outdoor use; its operating range is specified as -20 to $60^{\circ} \mathrm{C}$. Since its data acquisition and signal processing are done digitally its accuracy is not affected by a thermal coefficient. The Bartington Mag-03MCES100 fluxgate has a specified operating range from -40 to $70^{\circ} \mathrm{C}$, and its temperature-dependent offset is $<0.1 \mathrm{nT}^{\circ} \mathrm{C}^{-1}$. During normal use the temperature variation will be $<10^{\circ} \mathrm{C}$ over a measurement series, so the maximum error introduced by the fluxgate sensor is $1 \mathrm{nT}$. For the Measurement Computing USB-1608G DAQ a temperature coefficient is not provided, and its performance is specified while operating at $25^{\circ} \mathrm{C}$. The operational temperature range is between 0 and $70^{\circ} \mathrm{C}$. This DAQ converts the signals of the fluxgate and tilt sensor with 16-bit precision, while 14-bit precision would suffice the precision of $0.01 \mu \mathrm{T}$ for the fluxgate and $0.1^{\circ}$ for the tilt sensor. The temperature coefficient of the GPS, fluxgate, and DAQ devices can therefore safely be ignored.

The output of the ADXL335 tilt sensor, however, is affected by changes in temperature. We therefore tested the temperature dependance of this sensor using a superior tilt sensor, the ADXL354, which has a temperature sensor on board. We mounted these two chips together on a servo motor that rotated over $25^{\circ}$ at $10 \mathrm{~s}$ intervals. After five movements the servo returned to its initial position. To mimic temperature variations during normal use in the field as closely as possible, we put this setup in a windowsill in our laboratory and let it run for $128 \mathrm{~h}$. During these $5 \mathrm{~d}$ the temperature of the ADXL354 chip varied between 17.5 and $35.6^{\circ} \mathrm{C}$ (Fig. 5). It is evident that the $z$ axis of the ADXL335 chip especially suffers from changes in temperature, as its output varies between -2.6 and $+0.8 \%$ over $18.1^{\circ} \mathrm{C}$. The performance of the ADXL354 is superior to the chip that we used for the AnomalyMapper and is therefore preferable for future versions. It must be noted, however, that the tilt sensor chip as used in the AnomalyMapper is encapsulated in a blob of silicon putty. This material is a good thermal insulator and suppresses short-term temperature influences on the chip, such as cloudy or sunny spells, while measuring. Moreover, the temperature is not expected to change more than a few degrees Celsius during a series of measurements.

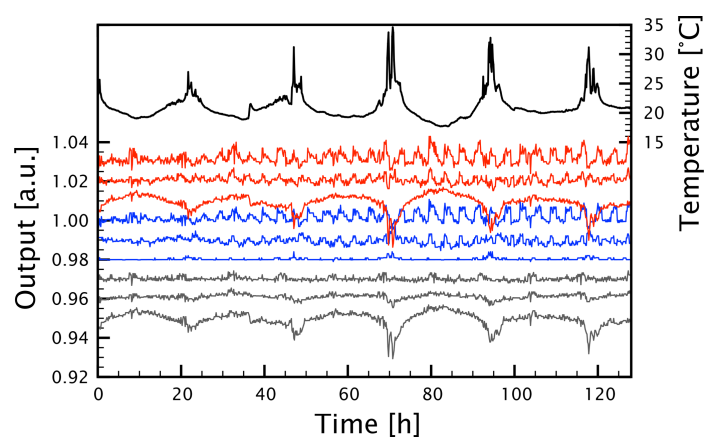

Figure 5. Temperature dependance of the tilt sensor readings. The temperature (in black) and the outputs of the ADXL335 (in red) and ADXL354 (in blue) were logged for $128 \mathrm{~h}$. The difference between the two sensors is in gray. For plotting purposes the output of the tilt sensors was normalized to the mean of the first 500 readings of the respective axis and then shifted to $1.03,1.02$, and 1.01 for the ADLX335 $x, y$, and $z$ axes; 1.00, 0.99, and 0.98 for the ADLX354 $x, y$, and $z$ axes; and $0.97,0.96$, and 0.95 for the difference between the two sensors ( $x, y$, and $z$ axes), respectively.

\subsection{Drift correction of tilt sensor}

Due to the thermal and possible mechanical drift of the ADXL335 tilt sensor as outlined above we cannot use the tilt sensor data in absolute terms. But, since the drift is limited over the course of a couple of hours and a small temperature range, we can use the mean value of all the data points in one measurement session to create an assumed true vertical vector for that measurement session. The assumption here is that over the course of a measurement session the AnomalyMapper is on average held upright. The rotation matrix from the true vertical vector initially established in the lab to the assumed true vertical vector from the mean value for each session is applied to all data points in that session, thus providing individual long-term drift correction for each measurement session.

\subsection{Choosing the reference target}

Choosing the proper reference target is paramount, and it is important to choose a point that can be seen from all measurement points. The GPS sensor that determines the location of the AnomalyMapper has an accuracy of $<1 \mathrm{~m}$. With a target at a distance of $200 \mathrm{~m}$, the maximum deviation in GPS position in the least favorable direction leads to an error in the bearing between the measurement and reference point locations of $<0.3^{\circ}$. Choosing the target even further away at $1 \mathrm{~km}$, for example, reduces this error to $<0.06^{\circ}$. In these calculations the GPS location of the reference point is considered accurate, as this location can be measured multiple times to improve the accuracy of the GPS location and can often easily be verified by satellite imagery. 


\section{Conclusions}

The AnomalyMapper is an accurate, easy to use, and lowcost device to measure local magnetic anomalies in volcanic terrain. Considering the reproducibility of the measurements during normal operation and the uncertainties associated with the different parts of the AnomalyMapper, it is capable of determining declinations and inclinations with an accuracy of at least $<0.5^{\circ}$. Data acquisition is quick: a grid of 110 points can be measured at three heights above the ground within $2.5 \mathrm{~h}$. By making use of a reference point on the ground to align the coordinate system of the AnomalyMapper to true north, east, and down, as well as a tilt sensor to rotate the fluxgate measurements to true vertical, the accuracy of the measurements is greatly improved. This experimental design also allows the AnomalyMapper to be used in all kinds of weather, except for very dense fog. The AnomalyMapper can be built for EUR $<1500$ if a commercial fluxgate sensor is at hand; otherwise, the total cost is EUR $\sim 3500$ for the entire setup.

Data availability. Both the raw data as measured by the AnomalyMapper and the processed data of the experiment on Mt. Etna are available in the Supplement to this paper.

Supplement. The supplement related to this article is available online at: https://doi.org/10.5194/gi-8-217-2019-supplement.

Author contributions. BMdG designed and built the instrument with the help of LVdG. LVdG prepared the paper with contributions from BMdG.

Competing interests. The authors declare that they have no conflict of interest.

Acknowledgements. Wout Krijgsman, Maartje van den Biggelaar, and Lynn Vogel helped acquire the data on Mt. Etna presented in this paper; Lynn Vogel processed the data, for which she is gratefully acknowledged.

Financial support. This research has been supported by the Dutch Research Council (Nederlandse Organisatie voor Wetenschappelijk Onderzoek, NWO; grant no. VENI 863.15.003).
Review statement. This paper was edited by Luis Vazquez and reviewed by four anonymous referees.

\section{References}

Baag, C., Helsley, C. E., Xu, S., and Lienert, B. R.: Deflection of paleomagnetic directions due to magnetization of the underlying terrain, J. Geophys. Res.-Sol. Ea., 100, 10013-10027, 1995.

Biggin, A. J., Perrin, M., and Dekkers, M. J.: A reliable absolute palaeointensity determination obtained from a non-ideal recorder, Earth Planet. Sc. Lett., 257, 545-563, 2007.

Castro, J. and Brown, L.: Shallow paleomagnetic directions from historic lava flows, Hawaii, Geophys. Res. Lett., 14, 1203-1206, 1987.

Coe, R. S., Jarboe, N. A., Le Goff, M., and Petersen, N.: Demise of the rapid-field-change hypothesis at Steens Mountain: The crucial role of continuous thermal demagnetization, Earth Planet. Sc. Lett., 400, 302-312, 2014.

de Groot, L. V., Biggin, A. J., Dekkers, M. J., Langereis, C. G., and Herrero-Bervera, E.: Rapid regional perturbations to the recent global geomagnetic decay revealed by a new Hawaiian record, Nat. Commun., 4, 2727, https://doi.org/10.1038/ncomms3727, 2013a.

de Groot, L. V., Mullender, T. A. T., and Dekkers, M. J.: An evaluation of the influence of the experimental cooling rate along with other thermomagnetic effects to explain anomalously low palaeointensities obtained for historic lavas of Mt Etna (Italy), Geophys. J. Int., 193, 1198-1215, 2013 b.

Greve, A., Hill, M., Turner, G., and Nilsson, A.: The geomagnetic field intensity in New Zealand: Palaeointensities from holocene lava flows of the tongariro Volcanic centre, Geophys. J. Int., 211, 814-830, 2017.

Nilsson, A., Holme, R., Korte, M., Suttie, N., and Hill, M.: Reconstructing Holocene geomagnetic field variation: new methods, models and implications, Geophys. J. Int., 198, 229-248, 2014.

Pavón-Carrasco, F. J., Osete, M. L., Torta, J. M., and De Santis, A.: A geomagnetic field model for the Holocene based on archaeomagnetic and lava flow data, Earth Planet. Sc. Lett., 388, 98-109, 2014.

Tanguy, J.-C. and Le Goff, M.: Distortion of the geomagnetic field in volcanic terrains: an experimental study of the Mount Etna stratovolcano, Phys. Earth Planet. In., 141, 59-70, 2004.

Valet, J.-P. and Soler, V.: Magnetic anomalies of lava fields in the Canary islands. Possible consequences for paleomagnetic records, Phys. Earth Planet. In., 115, 109-118, 1999. 